\title{
Atributos físicos do solo em sistemas de integração lavoura-pecuária-floresta
}

\author{
Paula C. R. Assis ${ }^{1}$, Luís F. Stone ${ }^{2}$, João C. Medeiros ${ }^{3}$, \\ Beata E. Madari ${ }^{4}$, Janína de M. Oliveira ${ }^{5}$ \& Flávio J. Wruck ${ }^{6}$
}

\section{Palavras-chave:}

qualidade física do solo

manejo do solo

pastagem degradada

\begin{abstract}
R E S U M O
Propôs-se, neste trabalho, determinar alterações nos atributos físicos do solo como resultado da implantação de sistemas de integração lavoura-pecuária-floresta (iLPF) e identificar aqueles adequados para detecção na mudança da qualidade física do solo (QFS). Amostras de solo foram coletadas nas camadas de 0-0,10 e 0,10-0,20 m em Nova Canãa do Norte, MT, e em Cachoeira Dourada, GO, em áreas sob iLPF com 1 (iLPF1) ou 3 (iLPF3) linhas de eucalipto e em áreas de pastagem recuperada e degradada. No iLPF1 as amostragens foram feitas na linha de árvores e a 2,5; 5,0 e 10,0 m dessa linha. No iLPF3, em Nova Canãa, as amostragens foram feitas na linha central e a 3,0; 6,0; 9,0 e 12,0 m dessa linha e, em Cachoeira Dourada, na linha central e a 1,5; 3,0; 4,5; 6,0 e 9,0 m dessa linha, com cinco repetições. Os sistemas iLPF promoveram melhoria na QFS em relação à pastagem degradada. Em Nova Canãa o iLPF1 propiciou melhor recuperação da QFS em relação à pastagem degradada, do que o iLPF3. A QFS nos sistemas iLPF variou com a posição de amostragem em relação à linha de árvores. A densidade e o arranjo poroso do solo na camada 0,10-0,20 $\mathrm{m}$ foram os principais responsáveis por discriminarem os sistemas integrados da pastagem degradada.
\end{abstract}

\section{Key words:}

soil physical quality

soil management

degraded pasture

\section{Physical attributes of soil in integrated crop-livestock-forest systems}

\begin{abstract}
A B S T R A C T
This study aimed to determine changes in soil physical attributes as result of implementation of integrated crop-livestock-forest (iCLF) systems and to identify atributes suitable for the detection of changes in soil physical quality (SFQ). Soil samples were collected in 0-0.10 and 0.10-0.20 m layers in Nova Canãa do Norte, MT, and Cachoeira Dourada, GO, in areas under iCLF with 1 (iCLF1) or 3 (iLCF3) lines of eucalyptus in areas of recovered and degraded pastures. In iCLF1, the samples were collected from the tree line and at 2.5, 5.0 and $10.0 \mathrm{~m}$ of this line. In iCLF3, in Nova Canãa do Norte, the samples were collected from the middle line and at 3.0; 6.0; 9.0 and $12.0 \mathrm{~m}$ of this line, and in Cachoeira Dourada, from the middle line and at $1.5 ; 3.0 ; 4.5 ; 6.0$ and $9.0 \mathrm{~m}$ of this line, with five replications. iCLF systems promoted improvement in SFQ regarding degraded pasture. In Nova Canãa do Norte, iCLF1 provided better recovery in SFQ, relative to degraded pasture, than iCLF3. The SFQ in iCLF systems varied with the sampling position relative to the line of trees. Bulk density and pore arrangement in the $0.10-0.20 \mathrm{~m}$ soil layer were mainly responsible for discriminating integrated systems from degraded pasture.
\end{abstract}

Protocolo 397-2013 - 10/12/2013 • Aprovado em 11/11/2014 • Publicado em 02/03/2015

${ }^{1}$ Pós-Graduação em Agronomia/UFG. Goiânia, GO. E-mail: paulacamyllaramos@gmail.com

${ }^{2}$ Embrapa Arroz e Feijão. Santo Antônio de Goiás, GO. E-mail: luis.stone@embrapa.br (Autor correspondente)

${ }^{3}$ Embrapa Arroz e Feijão. Santo Antônio de Goiás, GO. E-mail: medeiros.jc@gmail.com

${ }^{4}$ Embrapa Arroz e Feijão. Santo Antônio de Goiás, GO. E-mail: beata.madari@embrapa.br. Bolsista PQ do CNPq

${ }^{5}$ Pós-Graduação em Agronomia/UFG. Goiânia, GO. E-mail: janainamouraol@gmail.com

${ }^{6}$ Embrapa Arroz e Feijão. Santo Antônio de Goiás, GO. E-mail: flavio.wruck@embrapa.br 


\section{INTRODUÇÃo}

Os sistemas agrícolas que favorecem a qualidade do solo são aqueles que cultivam plantas intensivamente, de preferência de espécies diferentes, sem o revolvimento do solo (Vezzani \& Mielniczuk, 2009). A estrutura física do solo será tanto mais complexa quanto maiores forem a quantidade e a diversidade (quantidade, qualidade e frequência de aporte) da fitomassa disponibilizada ao sistema.

A intensificação da produção observada no sistema de integração lavoura-pecuária-floresta (iLPF) melhora as condições físicas, químicas e biológicas do solo; aumenta a ciclagem e a eficiência de utilização dos nutrientes; reduz custos de produção; diversifica e estabiliza a renda na propriedade rural e viabiliza a recuperação de áreas com pastagens degradadas (Alvarenga et al., 2010).

A elevação dos teores de matéria orgânica e a melhoria da qualidade física do solo pela introdução do sistema iLPF em áreas agrícolas com níveis adequados de fertilidade evidenciam o potencial deste sistema em reduzir não apenas o impacto ambiental das atividades produtivas mas também as emissões de gases de efeito estufa melhorando o aproveitamento da água e dos nutrientes do solo (Balbino et al., 2011). Na verdade, tem-se observado que nas áreas com sistemas integrados de cultivo a qualidade do solo é superior em relação às áreas com monocultivo (Maia et al., 2006; Aguiar, 2008; Jakelaitis et al., 2008; Loss et al., 2012).

O sistema iLPF está sendo considerado inovador e solução com alto potencial na recuperação de áreas degradadas e, assim, na supressão de desmatamento de novas áreas para

A.

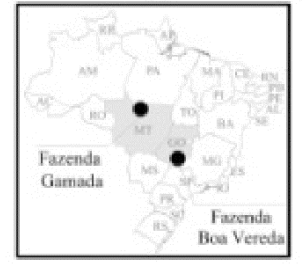

B.

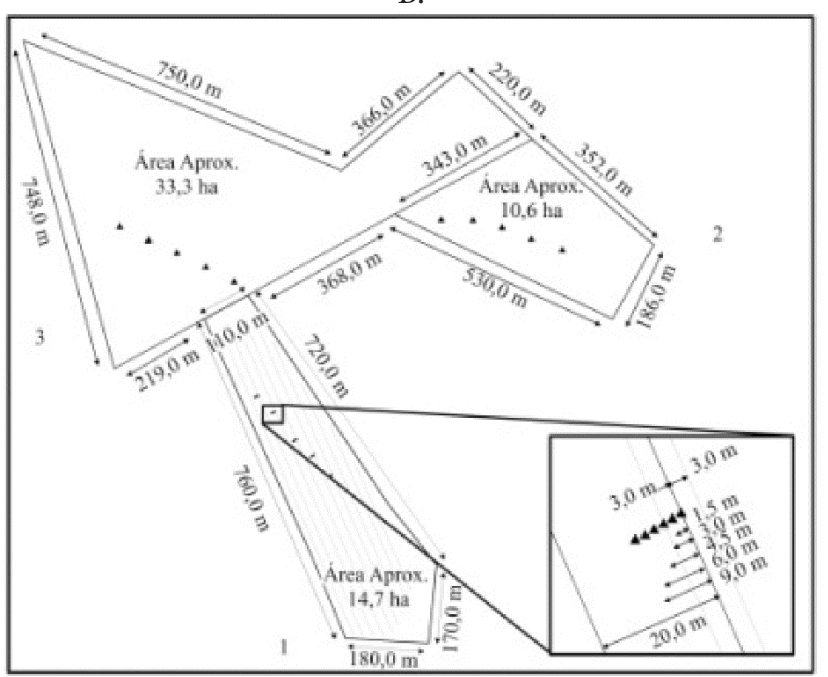

o uso na agropecuária porém seu impacto na qualidade do solo necessita de maiores estudos tornando imprescindível se estabelecer indicadores adequados para detecção das mudanças na qualidade do solo pela transformação de sistemas convencionais de uso do solo em sistemas iLPF.

Os atributos físicos do solo têm sido considerados por alguns autores (Araújo et al., 2007) indicadores das diferenças entre áreas com diferentes sistemas de uso da terra no Bioma Cerrado enquanto para outros (Carneiro et al., 2009) eles são os que apresentam as menores contribuições nesta discriminação.

Este trabalho objetivou determinar alterações nos atributos físicos do solo como resultado da implantação de sistemas de integração lavoura-pecuária-floresta e identificar aqueles adequados para a detecção na mudança da qualidade física do solo.

\section{Material e Métodos}

Para avaliar alterações nos atributos físicos do solo após a implantação de sistemas integrados de produção, foram estudadas duas áreas, uma na fazenda Gamada, em Nova Canaã do Norte, MT (10³8' 13” S, 5542' 32” W) e outra na fazenda Boa Vereda, em Cachoeira Dourada, GO (18² $27^{\prime} 43^{\prime \prime}$ S, 493' 58” W). Em Nova Canaã do Norte foram avaliados os sistemas iLPF com 1 (iLPF1) e 3 (iLPF3) linhas de eucalipto e, como referência, uma pastagem convencional degradada; em Cachoeira Dourada foram avaliados um sistema iLPF3 e uma pastagem convencional recuperada tendo, como referência, também uma pastagem convencional degradada (Figura 1).
C.

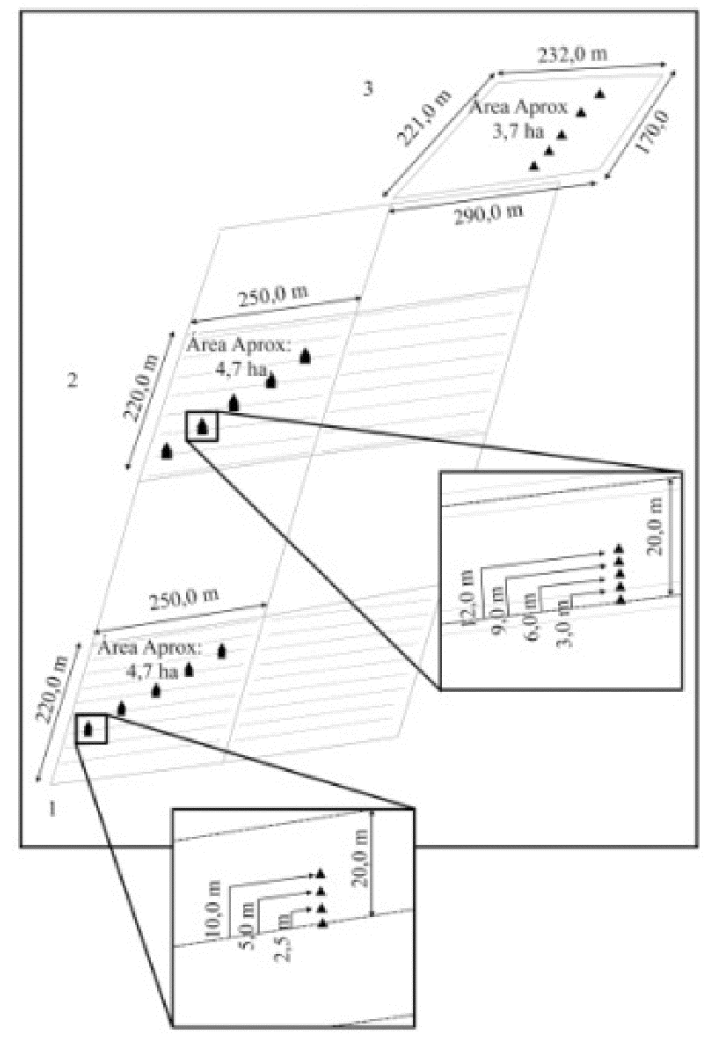

Figura 1. Localização das áreas estudadas (A); Fazenda Boa Vereda (B): 1. Área sob iLPF com três linhas de eucalipto, 2. Área sob pastagem degradada, 3. Área sob pastagem recuperada; Fazenda Gamada (C): 1 . Área sob iLPF com uma linha de eucalipto, 2. Área sob iLPF com três linhas de eucalipto, 3. Área sob pastagem degradada 
Em Nova Canaã do Norte os sistemas iLPF foram implantados em 2009, em um Latossolo Vermelho-Amarelo distroférrico, textura argilosa e em Cachoeira Dourada o iLPF3 foi implantado em 2008, em um Latossolo Vermelho acriférrico, textura argilosa. Em ambas as áreas a espécie de eucalipto usada foi Eucalyptus urograndis, no espaçamento de $2 \mathrm{~m}$ entre plantas na linha e $3 \mathrm{~m}$ entre linhas no conjunto de linhas triplas (renques) sendo de $20 \mathrm{~m}$ o espaçamento entre renques em Nova Canaã e de $14 \mathrm{~m}$ em Cachoeira Dourada.

Em Nova Canaã a abertura da área foi feita há mais de 10 anos, primeiro com o cultivo do arroz, depois com o de soja sendo implantada, posteriormente, a pastagem com Urochloa brizantha cv. Xaraés. Na implantação dos sistemas iLPF foi cultivado, no primeiro ano de plantio do eucalipto, o arroz, em plantio direto e, no segundo e terceiro anos, soja precoce e arroz precoce. No terceiro ano e após essas culturas, foi cultivado o milho consorciado com forrageira (Urochloa spp ou Panicum spp) o que também foi feito no quarto ano quando foi introduzida a pecuária de corte, com F1 resultante do cruzamento industrial da raça espanhola Rúbia Gallega x Nelore.

Em Cachoeira Dourada o iLPF3 foi implantado em área de pastagem com cerca de 30 anos, mediante o preparo do solo com grades aradora e niveladora, calagem e plantio de eucalipto e soja; no segundo ano foi cultivado o milho consorciado com $U$. brizantha cv. BRS Piatã e cv. Marandu e introduzida a pecuária de corte, com cruzamentos de Nelore. A área de pastagem recuperada é composta por capim-quicuio (Pennisetum clandestinum) e U. brizantha; nesta área também foi feita a calagem em 2008 e o solo foi preparado da mesma maneira que no iLPF3 para implantação da U. brizantha. A área de pastagem degradada, na qual não houve calagem, é composta por capim-quicuio ( $P$. clandestinum) e U. decumbens.

Coletas de amostras de solo nas áreas foram feitas em março de 2012, nas camadas de 0-0,10 e 0,10-0,20 m, para análise dos atributos físicos do solo; no iLPF1 as amostragens foram feitas na linha de árvores e a 2,5; 5,0 e 10,0 m dessa linha; na iLPF3, em Nova Canãa do Norte, as amostragens foram feitas na linha central e a 3,0 (linha mais externa), 6,0; 9,0 e 12,0 m dessa linha e, em Cachoeira Dourada, na linha central e a 1,5, 3,0 (linha mais externa), 4,5; 6,0 e 9,0 m dessa linha (Figura 1). O número de repetições foi igual a cinco com duas subamostras em cada camada.

Foram coletadas amostras com estrutura deformada e não deformada; as amostras com estrutura deformada foram utilizadas para determinar a textura do solo pelo método da pipeta e a densidade de partículas (Dp), pelo método do balão volumétrico; as amostras não deformadas, coletadas em cilindros de 0,05 m de diâmetro e 0,05 $\mathrm{m}$ de altura, foram usadas para determinação da condutividade hidráulica saturada (Ko) em permeâmetro de carga constante, curva de retenção de água do solo e densidade do solo (Ds). A porosidade total (Pt) foi calculada pela equação: $\mathrm{Pt}=(1-\mathrm{Ds} / \mathrm{Dp})$, a microporosidade (Mip) pelo conteúdo de água retida na tensão de $6 \mathrm{kPa}$ e a macroporosidade (Map) obtida pela diferença entre a Pt e a Mip (EMBRAPA, 1997). A determinação das curvas de retenção de água foi feita pelo método da centrífuga (Freitas Júnior \& Silva, 1984) visando ajustá-las por meio de regressão não-linear utilizando-se o modelo matemático proposto por Genuchten (1980) dado por:

$$
\theta=\left(\theta_{\text {sat }}-\theta_{\text {res }}\right)\left[1+(\alpha h)^{\mathrm{n}}\right]^{-\mathrm{m}}+\theta_{\text {res }}
$$

em que:

$\theta, \theta_{\text {sat }}$ e $\theta_{\text {res }}$ - conteúdos de água do solo correspondentes, respectivamente, à tensão $h$, à saturação e à umidade residual, em kg kg-1

h - tensão matricial da água do solo, em $\mathrm{kPa}$

$\mathrm{n}$ e $\mathrm{m}(\mathrm{m}=1-1 / \mathrm{n})$ - parâmetros empíricos adimensionais de ajuste

a - parâmetro expresso em $\mathrm{kPa}^{-1}$

Com base nos parâmetros obtidos o índice $S$, tangente à curva característica de água no solo no ponto de inflexão, foi determinado segundo a equação (Dexter, 2004):

$$
\mathrm{S}=-\mathrm{n}\left(\theta_{\text {sat }}-\theta_{\text {res }}\right)\left[1+\frac{1}{\mathrm{~m}}\right]^{-(1+\mathrm{m})}
$$

A capacidade de aeração do solo (CAS) foi calculada segundo a relação (Reynolds et al., 2002):

$$
\mathrm{CAS}=\frac{\mathrm{Pt}-\mathrm{CC}}{\mathrm{Pt}}
$$

em que:

CC - capacidade de campo, considerada igual ao conteúdo de água no solo na tensão de $8 \mathrm{kPa}$, expressa em $\mathrm{m}^{3} \mathrm{~m}^{-3}$

A capacidade de água disponível (CAD) foi calculada pela diferença entre a CC e o conteúdo de água na tensão de $1500 \mathrm{kPa}$, considerado ponto de murcha permanente (PMP), multiplicada pela espessura da camada considerada.

Em cada camada estudada os dados relativos aos diferentes tratamentos, resultantes da combinação dos sistemas iLPF1 e iLPF3 com as posições de amostragem, em Nova Canãa do Norte e resultantes das posições de amostragem no iLPF3 mais a pastagem recuperada, em Cachoeira Dourada, foram comparados com os obtidos nas pastagens degradadas (referência), pelo teste t a 0,05 de significância. Foram realizadas, também, análises de correlação de Pearson entre as variáveis estudadas; além disto, realizou-se análise de componentes principais em cada localidade envolvendo todos os usos da terra e atributos em estudo considerandose, conjuntamente, as duas camadas a partir das quais o conjunto de dados em combinações lineares foi reduzido gerando escores dos componentes principais que explicam em torno de $80 \%$ da variação total, conforme recomendado por Cruz \& Regazzi (1994). Adicionalmente, efetuou-se a análise de agrupamento pelo método de Ward utilizandose, como medida de dissimilaridade, a distância euclidiana média. As análises foram feitas com auxílio do Programa $\mathrm{R}$ (R Development Core Team, 2011).

\section{Resultados e Discuss ão}

Em Nova Canãa do Norte, na camada de 0-0,10 m, em todas as posições amostradas do iLPF1, os valores da Ko, à exceção da linha de árvores, os da Map e os da Pt, foram maiores e os 
da Ds foram menores em relação aos observados na pastagem degradada (Tabela 1). Esses resultados condicionaram maior qualidade física do solo no sistema iLPF1, medida pelo índice $\mathrm{S}$ e pela CAS, em relação à pastagem. Contudo, os valores da CAS se situaram abaixo de 0,34 , que é considerado o valor que reflete boa qualidade física do solo, de acordo com Reynolds et al. (2002).

Considerando $S=0,045$ como limítrofe entre solo de boa qualidade estrutural e solo com tendência a se tornar degradado e $\mathrm{S} \leq 0,025$ como indicativo de solos inteiramente degradados fisicamente (Andrade \& Stone, 2009) verificase que, com exceção da posição relativa à linha de árvores, o sistema iLPF1 apresentou valores de S maiores que 0,045 enquanto o índice $S$ da pastagem foi menor que 0,025.

A Ko aumentou com o incremento da Map e diminuiu com o aumento da Mip e da Ds (Tabela 2). Este atributo é dependente do arranjo poroso do solo, que sofre a ação do pisoteio animal e das práticas agrícolas. Na camada de 0,10-0,20 m observase redução da Ko com o distanciamento da linha das árvores no iLPF1. Este efeito pode ser relacionado à compactação do solo provocada pelo tráfego de máquinas ocorrido na fase das culturas anuais de grãos; já na camada $0-0,10 \mathrm{~m}$, a atividade biológica elevada, somada ao desenvolvimento radicular da pastagem, pode acelerar o processo de resiliência do solo e, consequentemente, recuperar sua estrutura aumentando a Ko. O índice $S$ e a CAS também estão altamente relacionados com o arranjo poroso do solo apresentando correlação positiva com a Ko, Map e Pt e negativa com a Ds (Tabela 2).

No iLPF3, em todas as posições os valores de Ko e da Map não se diferenciaram significativamente dos da pastagem (Tabela 1); contudo, a Ds foi menor e a Pt maior do que na
Tabela 2. Coeficiente de correlação (r) entre atributos do solo nas camadas de 0-0,10 e 0,10-0,20 m, em Nova Canãa do Norte, MT

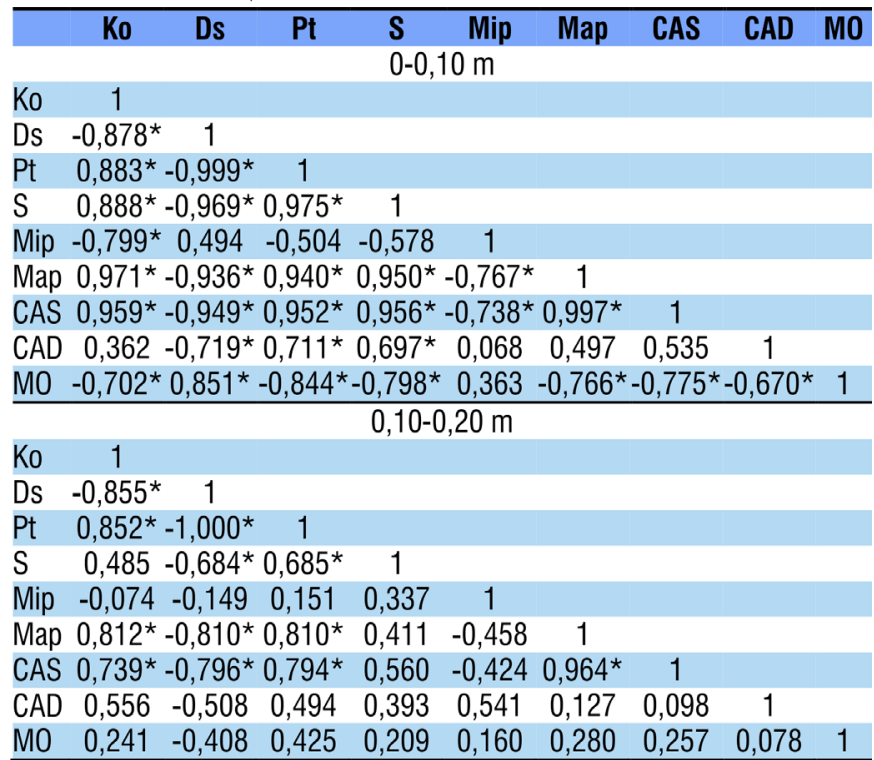

Valor de $r$ seguido de asterisco é significativo a 0,05 de probabilidade

Ko: condutividade hidráulica do solo; Ds: densidade do solo; Pt: porosidade total; Mip: microporosidade; Map: macroporosidade; S: índice S; CAS: capacidade de aeração do solo; CAD: capacidade de água disponível no solo; MO: matéria orgânica

pastagem com exceção das posições relativas a 6 e $12 \mathrm{~m}$ da linha central do renque de árvores, o que resultou em maiores valores do índice $S$ no sistema integrado, com exceção da posição 6,0 m, porém os valores de CAS não diferiram entre este sistema e a pastagem.

De maneira geral, a CAD no solo sob pastagem degradada foi menor que sob os iLPFs, possivelmente em razão da sua

Tabela 1. Valores médios da condutividade hidráulica saturada (Ko), densidade, relações de porosidade, índice S, capacidade de aeração do solo (CAS) e capacidade de água disponível (CAD) de acordo com os sistemas, posições e camadas estudadas, em Nova Canaã do Norte, MT, 2012

\begin{tabular}{|c|c|c|c|c|c|c|c|c|c|}
\hline \multirow{4}{*}{ Sistema } & \multirow{4}{*}{$\begin{array}{l}\text { Posição' } \\
\text { (m) }\end{array}$} & \multicolumn{8}{|c|}{ Camada (m) } \\
\hline & & $0-0,10$ & $0,10-0,20$ & $0-0,10$ & $0,10-0,20$ & $0-0,10$ & $0,10-0,20$ & $0-0,10$ & $0,10-0,20$ \\
\hline & & \multirow{2}{*}{\multicolumn{2}{|c|}{$\begin{array}{c}\mathrm{Ko}_{0} \\
\mathrm{~cm} \mathrm{~h}^{-1}\end{array}$}} & \multirow{2}{*}{\multicolumn{2}{|c|}{$\begin{array}{c}\text { Densidade do solo } \\
\mathrm{Mg} \mathrm{m}^{-3}\end{array}$}} & Micr & sidade & \multicolumn{2}{|c|}{ Macroporosidade } \\
\hline & & & & & & & & & \\
\hline \multirow{4}{*}{ iLPF1 } & 0 & 6,0 & $17,0^{*}$ & $1,26^{\star}$ & $1,18^{*}$ & 0,410 & 0,340 & $0,115^{\star}$ & $0,216^{*}$ \\
\hline & 2,5 & $15,3^{*}$ & 4,7 & $1,14^{*}$ & $1,34^{*}$ & 0,394 & $0,383^{*}$ & $0,176^{*}$ & 0,111 \\
\hline & 5,0 & $10,6^{\star}$ & 7,1 & $1,21^{*}$ & $1,32^{*}$ & 0,397 & $0,374^{*}$ & $0,149 *$ & $0,130^{*}$ \\
\hline & 10,0 & $10,2^{\star}$ & 5,5 & $1,17^{*}$ & $1,37^{*}$ & 0,394 & $0,373^{*}$ & $0,163^{*}$ & 0,110 \\
\hline \multirow{5}{*}{ iLPF3 } & 0 & 3,7 & $11,1^{*}$ & $1,25^{\star}$ & $1,19 *$ & $0,435^{*}$ & $0,403^{*}$ & 0,093 & $0,148^{*}$ \\
\hline & 3,0 & 4,8 & $14,5^{\star}$ & $1,24^{\star}$ & $1,21^{*}$ & 0,418 & $0,379^{*}$ & 0,116 & $0,163^{\star}$ \\
\hline & 6,0 & 1,5 & 3,8 & 1,35 & $1,31^{*}$ & 0,422 & $0,382^{*}$ & 0,068 & $0,125^{\star}$ \\
\hline & 9,0 & 1,3 & $9,4^{*}$ & $1,29 *$ & 1,30 * & $0,431^{*}$ & $0,418^{*}$ & 0,081 & 0,093 \\
\hline & 12,0 & 0,9 & 3,2 & 1,32 & $1,32^{*}$ & 0,424 & $0,375^{*}$ & 0,079 & $0,127^{*}$ \\
\hline \multirow[t]{2}{*}{ Pastagem } & & 2,3 & 2,5 & 1,39 & 1,47 & 0,404 & 0,354 & 0,073 & 0,092 \\
\hline & & \multicolumn{2}{|c|}{$\begin{array}{l}\text { Porosidade total } \\
\qquad \mathrm{m}^{3} \mathrm{~m}^{-3}\end{array}$} & \multicolumn{2}{|c|}{ Índice S } & \multicolumn{2}{|c|}{ CAS } & \multicolumn{2}{|c|}{$\begin{array}{l}\text { CAD } \\
\mathrm{mm}\end{array}$} \\
\hline \multirow{4}{*}{ iLPF1 } & 0 & $0,524^{*}$ & $0,556^{*}$ & $0,035^{\star}$ & $0,057^{*}$ & $0,24^{*}$ & 0,40 * & 9,6 & 8,0 \\
\hline & 2,5 & $0,569 *$ & $0,494^{*}$ & 0,049 * & $0,037^{*}$ & $0,33^{*}$ & 0,26 & $10,5^{\star}$ & 8,3 \\
\hline & 5,0 & $0,545^{\star}$ & $0,504^{\star}$ & $0,046 *$ & $0,036^{\star}$ & $0,28 *$ & $0,28^{*}$ & $9,9 *$ & 8,0 \\
\hline & 10,0 & $0,557^{*}$ & $0,484^{*}$ & $0,048^{*}$ & $0,033^{*}$ & 0,31 * & 0,25 & $10,9^{*}$ & 7,7 \\
\hline \multirow{5}{*}{ iLPF3 } & 0 & 0,528 * & $0,552^{\star}$ & $0,037^{\star}$ & $0,050^{*}$ & 0,20 & $0,34^{*}$ & $10,5^{\star}$ & $9,6^{*}$ \\
\hline & 3,0 & $0,533^{*}$ & $0,542^{*}$ & $0,042^{*}$ & $0,061^{*}$ & 0,24 & $0,33^{*}$ & $10,9 *$ & $11,6^{*}$ \\
\hline & 6,0 & 0,490 & $0,507^{\star}$ & 0,028 & $0,042^{\star}$ & 0,16 & $0,27^{*}$ & $9,9^{\star}$ & 8,0 \\
\hline & 9,0 & $0,512^{*}$ & $0,511^{*}$ & 0,030 * & $0,038^{*}$ & 0,18 & 0,19 & $10,2^{*}$ & 10,3 \\
\hline & 12,0 & 0,503 & $0,503^{\star}$ & 0,031 * & $0,036^{\star}$ & 0,18 & $0,27^{\star}$ & $10,0^{*}$ & 8,0 \\
\hline Pastagem & & 0,477 & 0,446 & 0,024 & 0,025 & 0,16 & 0,22 & 8,7 & 7,9 \\
\hline
\end{tabular}

Médias nas colunas seguidas de asterisco diferem da pastagem pelo teste t a 0,05; ${ }^{1}$ Em relação à linha de árvores, no caso do sistema iLPF1 e em relação à linha central do renque de árvores, no caso do sistema iLPF3 
maior Ds uma vez que a correlação entre esses dois atributos foi significativa e negativa (Tabelas 1 e 2). A CAD depende do PMP, que é determinado primariamente pelo seu teor de argila, que não é grandemente afetado pelo manejo do solo, e da CC, que é definida por interação complexa do teor de argila, densidade e matéria orgânica do solo (Reynolds et al., 2002).

Na camada de 0,10-0,20 m, nos dois iLPFs e em todas as posições, os valores da Pt foram maiores, os da Ds foram menores e os da CAD, com exceção da posição relativa à linha central do renque de árvores e a linha externa do iLPF3, não diferiram em relação aos observados na pastagem degradada (Tabela 1). Referidos resultados refletiram em maiores valores da qualidade física do solo medida pelo índice S. A CAS também apresentou, de maneira geral, maiores valores nos sistemas integrados com exceção das posições 2,5 e 10,0 m, na iLPF1 e da posição 9,0 m, na iLPF3.

Considerando as duas camadas estudadas, a análise de componentes principais mostrou que a variância acumulada nos primeiros dois componentes foi de $78,1 \%$, tendo o primeiro componente explicado $42,7 \%$ da variância total e o segundo 35,4\%. A Ds, Map, Pt, índice S e CAS na camada superficial (Tabela 3) propiciaram altos índices de correlação com o primeiro componente principal $(\mathrm{r}>|0,80|)$ e foram responsáveis por discriminar o sistema iLPF1 em relação aos demais. A Ds, Mip e Pt na camada 0,10-0,20 m apresentaram alta correlação com o segundo componente principal e discriminaram a pastagem dos demais sistemas.

O dendrograma resultante da análise de agrupamento ilustra a formação de dois grandes grupos (Figura 2), o primeiro composto pelo iLPF1 nas posições 2,5, 5,0 e 10,0 m da linha de árvores e o segundo pelos demais tratamentos. O segundo grupo apresentou, ainda, dois subgrupos, um formado pelo iLPF1, na posição da linha de árvores, e pelo iLPF3, na linha central e na linha externa do renque, e outro pela pastagem convencional e as demais posições do iLPF3.

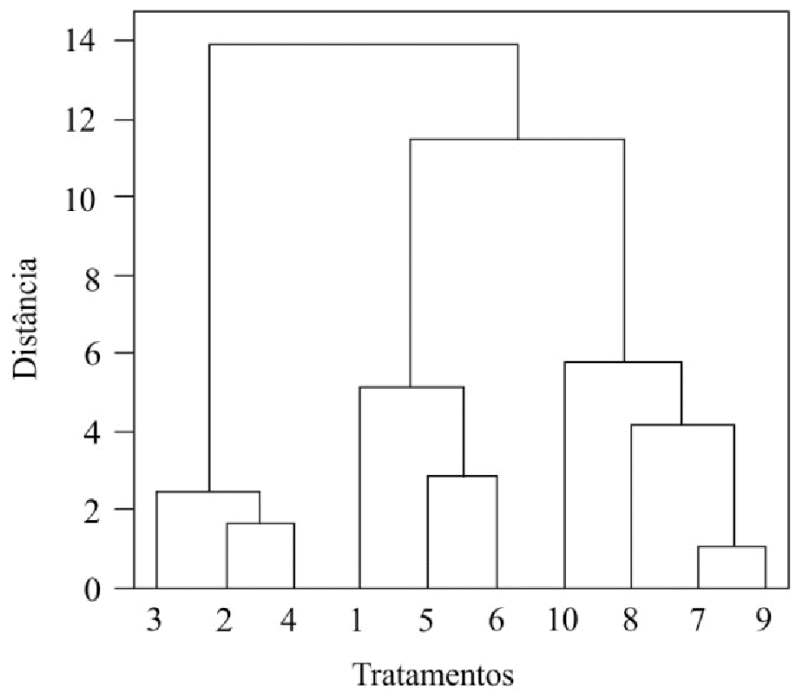

Figura 2. Dendrograma de agrupamento dos tratamentos na camada de 0-0,20 m, em Nova Canaã do Norte, MT. 1, 2, 3 e 4: iLPF1 e amostragem na linha de árvores e a 2,5; 5,0 e 10,0 m dessa linha, respectivamente; 5, 6, 7, 8 e 9: iLPF3 e amostragem na linha central e a 3,0; 6,0; 9,0 e 12,0 $\mathrm{m}$ dessa linha, respectivamente; 10: pastagem degradada
Tabela 3. Correlação entre cada componente principal e atributos físicos das camadas 0-0,10 e 0,10-0,20 m

\begin{tabular}{|c|c|c|c|c|}
\hline \multirow{2}{*}{ Atributos $^{1}$} & $\mathrm{CP}^{2}$ & CP2 & CP1 & $\overline{C P 2}$ \\
\hline & \multicolumn{2}{|c|}{$0-0,10 \mathrm{~m}$} & \multicolumn{2}{|c|}{$0,10-0,20 \mathrm{~m}$} \\
\hline & \multicolumn{4}{|c|}{ Nova Canãa do Norte } \\
\hline $\mathrm{K}_{0}\left(\mathrm{~cm} \mathrm{~h}^{-1}\right)$ & 0,79 & 0,56 & 0,50 & $-0,76$ \\
\hline Densidade do solo $\left(\mathrm{Mg} \mathrm{m}^{-3}\right)$ & $-0,94$ & $-0,27$ & $-0,48$ & 0,86 \\
\hline Microporosidade $\left(\mathrm{m}^{3} \mathrm{~m}^{-3}\right)$ & $-0,41$ & $-0,76$ & $-0,04$ & $-0,85$ \\
\hline Macroporosidade $\left(\mathrm{m}^{3} \mathrm{~m}^{-3}\right)$ & 0,86 & 0,50 & 0,45 & $-0,70$ \\
\hline Porosidade total $\left(\mathrm{m}^{3} \mathrm{~m}^{-3}\right)$ & 0,94 & 0,27 & 0,47 & $-0,86$ \\
\hline Índice S & 0,92 & 0,32 & 0,47 & $-0,11$ \\
\hline CAS & 0,89 & 0,45 & 0,50 & $-0,65$ \\
\hline \multirow[t]{2}{*}{$\mathrm{CAD}(\mathrm{mm})$} & 0,69 & $-0,11$ & 0,16 & $-0,58$ \\
\hline & \multicolumn{4}{|c|}{ Cachoeira Dourada } \\
\hline $\mathrm{Ko}_{0}\left(\mathrm{~cm} \mathrm{~h}^{-1}\right)$ & 0,38 & $-0,82$ & 0,31 & 0,62 \\
\hline Densidade do solo $\left(\mathrm{Mg} \mathrm{m}^{-3}\right)$ & $-0,76$ & 0,57 & 0,93 & 0,34 \\
\hline Microporosidade $\left(\mathrm{m}^{3} \mathrm{~m}^{-3}\right)$ & $-0,46$ & 0,72 & $-0,58$ & $-0,54$ \\
\hline Macroporosidade $\left(\mathrm{m}^{3} \mathrm{~m}^{-3}\right)$ & 0,69 & $-0,71$ & 0,82 & 0,46 \\
\hline Porosidade total $\left(\mathrm{m}^{3} \mathrm{~m}^{-3}\right)$ & 0,78 & $-0,56$ & 0,93 & 0,33 \\
\hline Índice S & 0,92 & $-0,06$ & 0,69 & 0,44 \\
\hline CAS & 0,77 & $-0,63$ & 0,84 & 0,52 \\
\hline $\mathrm{CAD}(\mathrm{mm})$ & 0,38 & 0,72 & 0,66 & 0,02 \\
\hline
\end{tabular}

${ }^{1}$ Ko - Condutividade hidráulica saturada, CAS - Capacidade de aeração do solo, CAD Capacidade de água disponível; ${ }^{2} \mathrm{CP} 1$ - Primeiro componente principal; CP2 - Segundo componente principal

O comportamento diferenciado dos atributos do solo nos diferentes sistemas pode estar relacionado com a rotação entre lavouras e pastagem e com a distribuição dos animais nas áreas. A compactação do solo que ocorre pelo pisoteio animal durante a fase de pastagem na rotação pode ser revertida quando da semeadura das lavouras, o que não ocorre na pastagem permanente. Macedo (2009) relatou que os sistemas integrados aumentam a estabilidade dos agregados e a taxa de infiltração de água e diminuem a densidade do solo e a compactação em relação a sistemas exclusivos, corroborando os resultados deste trabalho.

É provável que a concentração dos animais seja a causa dos teores mais elevados de matéria orgânica (MO) em determinadas posições de amostragem (Tabela 4) e do efeito deletério nos atributos físicos da camada superficial do solo nessas posições, uma vez que houve correlações negativas entre $\mathrm{MO}$ e Ko, Pt, Map, índice S, CAS e CAD e positiva entre ela e a Ds (Tabela 2). Na camada de $0,10-0,20 \mathrm{~m}$ as correlações entre $\mathrm{MO}$ e os atributos físicos do solo não foram significativas (Tabela 2).

Segundo Carvalho et al. (2005), em áreas de concentração dos animais houve comprometimento das propriedades físicas do solo; entretanto se encontrou, nessas áreas, maior disponibilidade de nutrientes devido à maior deposição de urina e fezes, havendo compensação química e não reduzindo a produtividade de soja e milho.

Não é previsto encontrar compactação do solo por pisoteio animal abaixo de $0,10 \mathrm{~m}$ de profundidade, em áreas manejadas em sistema plantio direto (Conte et al., 2011). O fato do pisoteio ocorrer na superfície do solo entre 0 e 0,10 $\mathrm{m}$, facilita ações para restabelecer a condição prévia do solo por agentes naturais e por operações de semeadura, como o preparo localizado na linha.

Em Cachoeira Dourada, na camada de 0-0,10 m, o valor da Ko sob pastagem não diferiu significativamente dos observados na iLPF, com exceção da posição relativa à linha central do renque de árvores (Tabela 5). Os valores da Ds, Mip, 
Tabela 4. Valores médios da matéria orgânica do solo $\left(\mathrm{g} \mathrm{kg}^{-1}\right)$ de acordo com os sistemas, posições e camadas estudadas, em Nova Canãa do Norte, MT, e Cachoeira Dourada, GO, 2012

\begin{tabular}{|c|c|c|c|}
\hline \multirow{2}{*}{ Sistema } & \multirow{2}{*}{$\begin{array}{c}\text { Posição }{ }^{1} \\
\text { (m) }\end{array}$} & \multicolumn{2}{|c|}{ Camada (m) } \\
\hline & & $0-0,10$ & $0,10-0,20$ \\
\hline \multicolumn{4}{|c|}{ Nova Canãa do Norte-MT } \\
\hline \multirow{4}{*}{ iLPF1 } & 0 & 29,8 & 21,1 \\
\hline & 2,5 & $26,8^{*}$ & 17,8 \\
\hline & 5,0 & 29,7 & 20,4 \\
\hline & 10,0 & $25,6^{\star}$ & 20,4 \\
\hline \multirow{5}{*}{ iLPF3 } & 0 & 27,1 & 22,2 \\
\hline & 3,0 & 28,9 & 19,9 \\
\hline & 6,0 & 32,4 & 20,3 \\
\hline & 9,0 & 30,1 & 21,1 \\
\hline & 12,0 & 32,1 & 22,2 \\
\hline \multicolumn{2}{|l|}{ Pastagem $^{3}$} & 31,3 & 19,8 \\
\hline \multicolumn{4}{|c|}{ Cachoeira Dourada-G0 } \\
\hline \multirow{6}{*}{ iLPF3 } & 0 & $35,1^{\star}$ & 32,3 \\
\hline & 1,5 & $36,1^{*}$ & 33,7 \\
\hline & 3,0 & $34,2^{\star}$ & 31,4 \\
\hline & 4,5 & $34,3^{\star}$ & 30,4 \\
\hline & 6,0 & 34,0 * & 31,7 \\
\hline & 9,0 & $34,2^{\star}$ & 30,4 \\
\hline Pastagem rec ${ }^{2}$ & & 46,8 & 36,6 \\
\hline Pastagem $^{3}$ & & 44,3 & 34,5 \\
\hline
\end{tabular}

Médias nas colunas seguidas de asterisco diferem da pastagem pelo teste t a $5 \%$; ${ }^{1}$ Em relação à linha de árvores, no caso do sistema iLPF1 e em relação à linha central do renque de árvores, no caso do sistema iLPF3; ${ }^{2}$ Pastagem recuperada; ${ }^{3}$ Pastagem degradada

Map e Pt sob pastagem degradada também não diferiram dos observados no iLPF porém a Ds e a Mip foram maiores e os demais atributos menores em relação à pastagem recuperada (Tabela 5) o que se supunha visto que ter sido exposta a práticas para recuperação, envolvendo preparo do solo.

Em razão desses resultados a CAS foi maior na pastagem recuperada em relação à pastagem degradada a qual, por sua vez, não diferiu do iLPF em nenhuma das posições de amostragem; contudo, quando se considera a qualidade do solo avaliada pelo índice S, o iLPF, em todas as posições, com exceção da linha central do renque de árvores, e a pastagem recuperada apresentaram maiores valores de $S$ que a pastagem degradada; isto ocorreu apesar da MO ser menor no iLPF do que nas pastagens (Tabela 4) devido, possivelmente, à movimentação do solo quando da implantação das culturas.

O solo sob iLPF apresentou, em todas as posições amostradas, maiores valores de CAD que o sob pastagem degradada a qual, por sua vez, não diferiu da pastagem recuperada quanto a este atributo (Tabela 5).

Como em Nova Canãa do Norte, a Ko aumentou com a Map e diminuiu com o aumento da Ds e da Mip, além de se correlacionar positivamente com a MO (Tabela 6). O índice $\mathrm{S}$ também apresentou correlação positiva com a Pt e CAS e negativa com a Ds.

Na camada de 0,10-0,20 m a Ko na linha central do renque de árvores e a 1,5 $\mathrm{m}$ dessa linha foi maior que na pastagem degradada, não diferindo dela nas demais posições de amostragem. Em todas as posições amostradas no iLPF3 e na pastagem recuperada, os valores da Map e Pt foram maiores e os da Ds foram menores em relação aos observados na pastagem degradada (Tabela 5). Esses resultados condicionaram maiores valores da qualidade física do solo, tanto medida pelo índice $S$ como pela CAS. A área sob pastagem recuperada e o iLPF em duas posições de amostragem também apresentaram maiores valores de $\mathrm{CAD}$ em relação à pastagem degradada.

Similarmente à Nova Canãa do Norte, nesta camada a MO no iLPF não diferiu significativamente da verificada sob pastagem (Tabela 4) e não se correlacionou com os demais atributos do solo (Tabela 6).

Considerando as duas camadas de amostragem, a análise de componentes principais mostrou que a variância acumulada nos primeiros dois componentes foi de $80,8 \%$, tendo o primeiro componente explicado $50,5 \%$ da variância total e o segundo $30,3 \%$. O índice S na camada superficial e a Ds, Map, Pt e CAS

Tabela 5. Valores médios da condutividade hidráulica saturada (Ko), densidade, relações de porosidade, índice S, capacidade de aeração do solo (CAS) e capacidade de água disponível (CAD) de acordo com os sistemas, posições e camadas estudadas, em Cachoeira Dourada, GO, 2012

\begin{tabular}{|c|c|c|c|c|c|c|c|c|c|}
\hline \multirow{4}{*}{ Sistema } & \multirow{4}{*}{$\begin{array}{l}\text { Posição }{ }^{1} \\
\text { (m) }\end{array}$} & \multicolumn{8}{|c|}{ Camada (m) } \\
\hline & & $0-0,10$ & $0,10-0,20$ & $0-0,10$ & $0,10-0,20$ & $0-0,10$ & $0,10-0,20$ & $0-0,10$ & $0,10-0,20$ \\
\hline & & \multirow{2}{*}{\multicolumn{2}{|c|}{$\begin{array}{c}\mathrm{Ko}_{0} \\
\mathrm{~cm} \mathrm{~h}^{-1}\end{array}$}} & \multirow{2}{*}{\multicolumn{2}{|c|}{$\begin{array}{c}\text { Densidade do solo } \\
\mathrm{Mg} \mathrm{m}^{-3}\end{array}$}} & Micro & sidade & \multicolumn{2}{|c|}{ Macroporosidade } \\
\hline & & & & & & & & & \\
\hline \multirow{6}{*}{ iLPF3 } & 0 & $8,2^{*}$ & $38,0^{*}$ & 1,23 & $1,09^{*}$ & 0,442 & $0,392^{*}$ & 0,094 & $0,195^{*}$ \\
\hline & 1,5 & 18,7 & $38,0^{*}$ & 1,18 & $1,08 *$ & 0,432 & $0,377^{*}$ & 0,121 & $0,214^{*}$ \\
\hline & 3,0 & 13,0 & 29,0 & 1,18 & $1,12^{\star}$ & 0,438 & 0,424 & 0,118 & 0,152 \\
\hline & 4,5 & 9,8 & 22,4 & 1,20 & $1,11^{*}$ & 0,455 & $0,413^{*}$ & 0,092 & $0,167^{*}$ \\
\hline & 6,0 & 10,3 & 24,8 & 1,18 & $1,10^{*}$ & 0,454 & $0,388^{*}$ & 0,100 & $0,195^{\star}$ \\
\hline & 9,0 & 13,4 & 26,7 & 1,18 & $1,12^{*}$ & 0,446 & $0,409 *$ & 0,109 & $0,168^{\star}$ \\
\hline \multirow{3}{*}{$\begin{array}{l}\text { Pastagem rec }{ }^{2} \\
\text { Pastagem }^{3}\end{array}$} & & 21,5 & 20,1 & $1,11^{*}$ & $1,07^{*}$ & $0,419^{*}$ & $0,408^{*}$ & $0,162^{*}$ & $0,188^{*}$ \\
\hline & & 15,6 & 18,3 & 1,22 & 1,24 & 0,440 & 0,434 & 0,099 & 0,097 \\
\hline & & \multicolumn{2}{|c|}{$\begin{array}{l}\text { Porosidade total } \\
\mathrm{m}^{3} \mathrm{~m}^{-3}\end{array}$} & \multicolumn{2}{|c|}{ Índice S } & \multicolumn{2}{|c|}{ CAS } & \multicolumn{2}{|c|}{$\begin{array}{l}\text { CAD } \\
\mathrm{mm}\end{array}$} \\
\hline \multirow{6}{*}{ iLPF3 } & 0 & 0,536 & $0,588 *$ & 0,050 & $0,080 *$ & 0,20 & $0,36^{*}$ & $11,6^{*}$ & 10,0 \\
\hline & 1,5 & 0,554 & $0,591^{*}$ & $0,059^{*}$ & $0,074^{*}$ & 0,25 & 0,39 * & $11,9 *$ & 9,2 \\
\hline & 3,0 & 0,556 & $0,577^{*}$ & $0,061^{*}$ & $0,071^{*}$ & 0,24 & $0,32^{*}$ & $12,3^{*}$ & $10,4^{*}$ \\
\hline & 4,5 & 0,546 & $0,580^{*}$ & $0,057^{*}$ & $0,072^{*}$ & 0,20 & $0,32^{*}$ & $13,2^{*}$ & $10,8^{*}$ \\
\hline & 6,0 & 0,554 & $0,583^{*}$ & $0,058^{*}$ & $0,073^{*}$ & 0,21 & $0,36^{*}$ & $12,8^{*}$ & 9,3 \\
\hline & 9,0 & 0,555 & $0,577^{*}$ & $0,055^{\star}$ & $0,096^{*}$ & 0,23 & $0,33^{*}$ & $12,2^{\star}$ & 9,7 \\
\hline Pastagem rec & & $0,582^{\star}$ & $0,596^{\star}$ & $0,066^{\star}$ & $0,076^{\star}$ & $0,30^{\star}$ & $0,34^{*}$ & 10,8 & $11,0^{*}$ \\
\hline Pastagem & & 0,538 & 0,530 & 0,040 & 0,039 & 0,20 & 0,19 & 9,6 & 8,2 \\
\hline
\end{tabular}

Médias nas colunas seguidas de asterisco diferem da pastagem pelo teste t a 0,05; ${ }^{1}$ Em relação à linha central do renque de árvores; ${ }^{2}$ Pastagem recuperada; ${ }^{3 P a s t a g e m ~ d e g r a d a d a ~}$ 
Tabela 6. Coeficiente de correlação (r) entre atributos do solo nas camadas de 0-0,10 e 0,10-0,20 m, em Cachoeira Dourada, GO

\begin{tabular}{|c|c|c|c|c|c|c|c|c|}
\hline & $\overline{K o}$ & $\overline{D s}$ & $\overline{\mathrm{PT}}$ & Mip & Map & $\overline{\text { CAS }}$ & $\overline{\text { CAD }}$ & MO \\
\hline \multicolumn{9}{|c|}{$0-0,10 \mathrm{~m}$} \\
\hline Ko & 1 & & & & & & & \\
\hline Ds & $-0,712^{\star}$ & 1 & & & & & & \\
\hline $\mathrm{Pt}$ & 0,696 & $-0,998 *$ & 1 & & & & & \\
\hline S & 0,325 & $-0,808^{*}$ & $0,821 *$ & 1 & & & & \\
\hline Mip & $-0,855^{\star}$ & 0,617 & $-0,627$ & $-0,337$ & & & & \\
\hline Map & $0,852^{*}$ & $-0,916$ * & 0,921 * & $0,662-0,880$ * & 1 & & & \\
\hline CAS & $0,836^{*}$ & $-0,922^{*}$ & $0,930^{*}$ & $0,730^{*}-0,850^{*}$ & $0,988^{*}$ & 1 & & \\
\hline CAD & $-0,534$ & 0,000 & 0,009 & $0,509 \quad 0,594$ & $-0,299$ & $-0,189$ & 1 & \\
\hline MO & $0,725^{\star}$ & $-0,428$ & 0,409 & $-0,104-0,707^{*}$ & 0,612 & 0,498 & $-0,843^{\star}$ & 1 \\
\hline \multicolumn{9}{|c|}{$0,10-$} \\
\hline Ko & 1 & & & & & & & \\
\hline Ds & $-0,491$ & 1 & & & & & & \\
\hline $\mathrm{Pt}$ & 0,488 & $-1,000^{*}$ & 1 & & & & & \\
\hline S & 0,430 & $-0,762^{*}$ & $0,772^{*}$ & 1 & & & & \\
\hline Mip & $-0,687$ & $0,723^{*}$ & $-0,707^{\star}$ & $-0,498$ & & & & \\
\hline Мар & 0,626 & $-0,935^{\star}$ & $0,926^{\star}$ & $0,689-0,921^{*}$ & 1 & & & \\
\hline CAS & 0.667 & $-0,950^{\star}$ & $0,945^{\star}$ & $0,765^{\star}-0,860^{\star}$ & $0,976^{\star}$ & 1 & & \\
\hline CAD & $-0,022$ & $-0,676$ & 0,691 & $0,530-0,010$ & 0,386 & 0,452 & 1 & \\
\hline MO & $-0,219$ & 0,042 & $-0,043$ & $-0,415 \quad 0,021$ & $-0,029$ & $-0,177$ & $-0,086$ & 1 \\
\hline
\end{tabular}

Valor de $\mathbf{r}$ seguido de asterisco é significativo a 0,05 de probabilidade

Ko - condutividade hidráulica do solo; Ds - densidade do solo; Pt - porosidade total; Mip microporosidade; Map - macroporosidade; S - índice S; CAS - capacidade de aeração do solo; CAD - capacidade de água disponível no solo; MO - matéria orgânica

na camada 0,10-0,20 m (Tabela 3) propiciaram altos índices de correlação com o primeiro componente principal ( $r>|0,80|)$ e foram responsáveis por discriminar a pastagem degradada dos demais sistemas. No sistema iLPF3 e na pastagem recuperada os atributos físicos apresentaram valores mais favoráveis na camada 0,10-0,20 m, maximizando as diferenças em relação à pastagem degradada. A Ko na camada superficial apresentou alta correlação com o segundo componente principal e descriminou a pastagem recuperada do sistema iLPF.

O dendrograma resultante da análise de agrupamento ilustra a formação de três grupos (Figura 3), um constituído pela pastagem degradada, outro pela recuperada e outro pelos tratamentos de iLPF. Analisando o último grupo verifica-se que

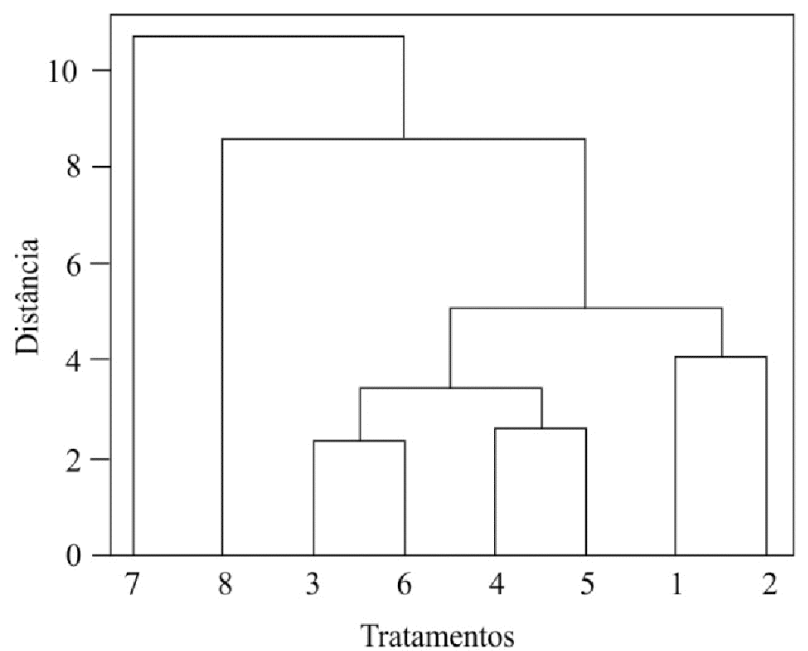

Figura 3 Dendrograma de agrupamento dos tratamentos na camada de 0-0,20 m em Cachoeira Dourada, GO. 1, 2, 3, 4, 5 e 6: iLPF3 e amostragem na linha central e a 1,5; 3,0; 4,5; 6,0 e 9,0 m dessa linha, respectivamente; 7: pastagem degradada; 8: pastagem recuperada o subgrupo formado pela linha central do renque de árvores e a posição 1,5 m desta linha estão mais afastados da pastagem recuperada enquanto o subgrupo formado pela posição 3,0 e 9,0 $\mathrm{m}$ da linha central do renque está mais próximo da pastagem recuperada.

\section{Conclusões}

1. Os sistemas de integração lavoura-pecuária-floresta promoveram melhoria na qualidade física do solo, em relação à pastagem degradada.

2. Em Nova Canãa do Norte o sistema iLPF com uma linha de eucalipto propiciou melhor recuperação da qualidade física do solo em relação a uma pastagem degradada, do que o sistema iLPF com três linhas de eucalipto.

3. A qualidade física do solo nos sistemas iLPF variou com a posição de amostragem em referência à linha de árvores.

4. A densidade e o arranjo poroso do solo na camada 0,10 $0,20 \mathrm{~m}$ foram os principais responsáveis por discriminarem os sistemas integrados da pastagem degradada.

\section{Agradecimentos}

Os autores agradecem às Dras Flávia A. Alcântara e Marina M. Morales, pelas sugestões técnicas, e ao $\mathrm{CNPq}$ projeto 562.621/2010-4 e EMBRAPA projeto 02.11.05.001, pelo suporte financeiro.

\section{Literatura Citada}

Aguiar, M. I. de. Qualidade física do solo em sistemas agroflorestais. Viçosa: UFV, 2008. 89p. Dissertação Mestrado

Alvarenga, R. C.; Silva, V. P. da; Gontijo Neto, M. M; Viana, M. C. M.; Vilela, L. Sistema Integração Lavoura-Pecuária-Floresta: Condicionamento do solo e intensificação da produção de lavouras. Informe Agropecuário, v.31, p.59-67, 2010.

Andrade, R. da S.; Stone, L. F. Índice S como indicador da qualidade física de solos do cerrado brasileiro. Revista Brasileira de Engenharia Agrícola e Ambiental, v.13, p.382-388, 2009. http:// dx.doi.org/10.1590/S1415-43662009000400003

Araújo, R.; Goedert, W. J.; Lacerda, M. P. C. Qualidade de um solo sob diferentes usos e sob Cerrado nativo. Revista Brasileira de Ciência do Solo, v.31, p.1099-1108, 2007. http://dx.doi.org/10.1590/S010006832007000500025

Balbino, L. C.; Martinez, G. B.; Galerani, P. R. Ações de transferência de tecnologia de sistemas de integração lavoura-pecuária-floresta. Planaltina: Embrapa Cerrados; Belém: Embrapa Amazônia Oriental, 2011. 52p.

Carneiro, M. A. C.; Souza, E. D. de; Reis, E. F. dos, Pereira, H. S.; Azevedo, W. R. de. Atributos físicos, químicos e biológicos de solo de Cerrado sob diferentes sistemas de uso e manejo. Revista Brasileira de Ciência do Solo, v.33, p.147-157, 2009. http://dx.doi. org/10.1590/S0100-06832009000100016

Carvalho, P. C. de F.; Anghinoni, I.; Moraes, A. de; Trein, C. R.; Flores, J. P C.; Cepik, C. T. C.; Levien, R.; Lopes, M. T. L.; Baggio, C.; Lang, C. R.; Sulc, R. M.; Pelissari, A. O estado da arte em integração lavoura-pecuária. In: Gottschall, C. S.; Silva, J. L. S.; Rodrigues, N. C. (org.). Produção animal: Mitos, pesquisa e adoção de tecnologia. Canoas: Ulbra, 2005. p.7-44. 
Conte, O.; Flores, J. P. C.; Cassol, L. C.; Anghinoni, I.; Carvalho, P. C. de F.; Levien, R.; Wesp, C. de L. Evolução de atributos físicos de solo em sistema de integração lavoura-pecuária. Pesquisa Agropecuária Brasileira, v.46, p.1301-1309, 2011. http://dx.doi. org/10.1590/S0100-204X2011001000026

Cruz, C. D.; Regazzi, A. J. Modelos biométricos aplicados ao melhoramento genético. Viçosa: UFV, 1994. 390p.

Dexter, A. R. Soil physical quality. Part 1. Theory, effects of soil texture, density, and organic matter, and effects on root growth. Geoderma, v.120, p.201-214, 2004. http://dx.doi.org/10.1016/j. geoderma.2003.09.004

EMBRAPA - Empresa Brasileira de Pesquisa Agropecuária. Centro Nacional de Pesquisas de Solos (Rio de Janeiro). Manual de métodos de análises de solo. 2.ed. Rio de Janeiro: EMBRAPA, 1997. 212p.

Freitas Júnior, E.; Silva, E. M. Uso da centrífuga para a determinação da curva de retenção de água no solo, em uma única operação. Pesquisa Agropecuária Brasileira, v.19, p.1423-1428, 1984.

Genuchten, M. T. van. A closed form equation for predicting the hydraulic properties of unsaturated soils. Soil Science Society of America Journal, v.44, p.892-898, 1980. http://dx.doi.org/10.2136/ sssaj1980.03615995004400050002x

Jakelaitis, A.; Silva, A. A. da; Santos, J. B. dos; Vivian, R. Qualidade da camada superficial de solo sob mata, pastagens e áreas cultivadas. Pesquisa Agropecuária Tropical, v.38, p.118-127, 2008.
Loss, A.; Pereira, M. G.; Beutler, S. J.; Perin, A.; Anjos, L. H. C. dos. Densidade e fertilidade do solo sob sistemas de plantio direto e de integração lavoura-pecuária no Cerrado. Revista Ciências Agrárias, v.55, p.260-268, 2012.

Macedo, M. C. M. Integração lavoura e pecuária: O estado da arte e inovações tecnológicas. Revista Brasileira de Zootecnia, v.38, p.133-146, 2009. http://dx.doi.org/10.1590/S151635982009001300015

Maia, S. M. F.; Xavier, F. A. da S.; Oliveira, T. S. de; Mendonça, E. de S.; Araújo Filho, J. A. de. Impactos de sistemas agroflorestais e convencional sobre a qualidade do solo no semi-árido cearense. Revista Árvore, v.30, p.837-848, 2006. http://dx.doi.org/10.1590/ S0100-67622006000500018

R Development Core Team. R: A language and environment for statistical computing. Vienna: R foundation for statistical computing, 2011. http://www.R-project.org. 25 Jan. 2011.

Reynolds, W. D.; Bowman, B. T.; Drury, C. F.; Tan, C. S.; Lu, X. Indicators of good soil physical quality: Density and storage parameters. Geoderma, v.110, p.131-146, 2002. http://dx.doi. org/10.1016/S0016-7061(02)00228-8

Vezzani, F. M.; Mielniczuk, J. Uma visão sobre a qualidade do solo. Revista Brasileira de Ciência do Solo, v.33, p.743-755, 2009. http:// dx.doi.org/10.1590/S0100-06832009000400001 